\title{
O USO DA TH APÓS A MENOPAUSA PROMOVE BENEFÍ́CIOS?
}

Otávio Celso Eluf Gebara ${ }^{1}$, Alesssandro Scapinelli ${ }^{2 \star}$, José Mendes Aldrighi ${ }^{3}$

Trabalho realizado na Faculdade de Ciências Médicas da Santa Casa de São Paulo, S. Paulo, SP

\author{
*Correspondência: \\ Rua Ernesto de Oliveira, $n^{\circ} 40$ - apto. 73 \\ Jardim Vila Mariana \\ São Paulo - SP \\ CEP: 04116170 \\ alescapinelli@uol.com.br
}

O uso da (TH) após menopausa ainda é motivo de muitas polêmicas, uma vez que as primeiras investigações observacionais tinham revelado significativa redução da mortalidade em suas usuárias quando comparadas com as não usuárias².

Entretanto, após a publicação do WHO (Women's Health Initiative $)^{3}$ constatou-se exatamente o contrário, ou seja, a $\mathrm{TH}$ se associava a uma maior morbimortalidade independente da faixa etária, o que culminou na orientação de que sua prescrição só deveria ser feita para curtos períodos e exclusivamente para sintomas menopausais ${ }^{4,5}$

A partir daí, muitas mulheres suspenderam abruptamente a $\mathrm{TH}$, resultando numa significativa piora na qualidade de vida ${ }^{6}$.

Por isso, tentando esclarecer a questão sobre o efeito da $\mathrm{TH}$ em diferentes idades foram publicadas duas metanálises envolvendo estudos randomizados, cujos resultados surpreendentemente constataram redução do número de eventos cardiovasculares e da mortalidade em mulheres mais jovens, mas não nas mais idosas ${ }^{6}$.

Neste estudo de 2009, Salpeter et al. decidiram elucidar a questão do uso da TH após a menopausa, a partir de um modelo matemático, em que avaliaram a relação custo-efetividade em diferentes faixas etárias.

Os resultados mostraram expressivo avanço na hipótese da janela de oportunidade para o uso da $\mathrm{TH}$, pois comparou dois grupos: mulheres mais jovens após a menopausa (até 59 anos) e mulheres mais idosas ( $>60$ anos). 0 modelo matemático se baseou em metanálise de estudos clínicos randomizados e controlados realizados até março de 2008, sendo avaliado - QALY (quality-ajusted-life years) que representa a relação custo-benefício da TH sobre qualidade de vida de mulheres após a menopausa.

Assim, o estudo concluiu que havia bom custo-benefício no uso da TH no grupo mais jovem e que isso não ocorria no grupo mais idoso; de fato, o grupo jovem apresentou ganho de 1,49 QUALYS a um custo de US\$2.438 (valores < US\$ 10.000 são significantemente custo-efetivos). Por outro lado, no grupo mais idoso, o ganho de QUALY foi de apenas 0,11 , a um custo de US\$27.953. Além disso, nos primeiros nove anos de tratamento constatou-se piora na qualidade de vida das mulheres mais idosas e somente a partir daí observou-se melhora. No grupo jovem, a melhora de $Q A L Y$ foi evidenciada teoricamente com períodos de tratamento que se estendeu por até 30 anos.

Esses mesmos autores já haviam demonstrado em metanálise de estudos clínicos controlados a ocorrência de redução da mortalidade em mulheres mais jovens que fizeram uso de $\mathrm{TH}$, sem qualquer benefício para o grupo que iniciou a $\mathrm{TH}$ tardiamente.

Do exposto se depreende que os resultados obtidos nesse estudo coincidem com os relatados anteriormente nos desenhos observacionais - que tinham incluído mulheres mais jovens e que iniciaram a TH logo após a menopausa -, ou seja, demonstraram melhora na qualidade de vida e redução de eventos cardíacos. Ademais, corrobora os resultados obtidos de estudos clínicos controlados que avaliaram mulheres mais idosas, como o Women's Health Initiative, que não demonstrou redução de mortalidade.

Baseados em todos esses estudos, as diretrizes brasileiras e internacionais têm recomendado o uso de TH em mulheres com sintomas menopausais, como ondas de calor, insônia, secura vaginal, a partir do estágio da transição menopausal e desaconselham seu início muitos anos após a idade da menopausa.

Referências

1. Salpenter SR, Buckley NS, Lui H, Salpeter EE. The Cost-effectiveness of Hormone therapy in younger and older postmenopausal Women. Am J Med. $2009 ; 122(1): 42-52$

1. Livre-docente da Divisão Clínica de Cardiogeriatria do InCor da Faculdade de Medicina da Universidade de São Paulo - USP, São Paulo, SP

2. Pós-graduando do Departamento de Obstetrícia Ginecologia da Faculdade de Ciências Médicas da Santa Casa de São Paulo, São Paulo, SP

3. Professor Titular da Clínica de Ginecologia Endócrina, Climatério e Anticoncepção (GECLAN) da Faculdade de Ciências Médicas da Santa Casa de São Paulo, São Paulo, SP 
2. Grodstein F, Mason JE, Colditz GA, Willett WC, Speizer FE, Stampfer MJ, et al. A prospective, Observational study of postmenopausal hormone therapy and primary preventionof cardiovascular disease. Ann Intern Med. 2000;133(12):933-41.

3. Rossouw JE, Anderson GL, Prentice RL, LaCroix AZ, Kooperberg C, Stefanick $\mathrm{ML}$, Jackson RD, et al. Risks and benefits of estrogen plus progestin in healthy postmenopausal women: principal results from the Women's Health Initiative randomized controlled trial. JAMA. 2002;288(3):312-33.
4. Majumdar SR, Almasi EA, Stafford RS. Promotion and prescribing of hormone therapy after report of harm by the Women's Health Initiative. JAMA. 2004;292(16): 1983-8

5. Salpeter SR, Walsh JM, Greyber E, Ormiston TM, Salpeter EE. Mortality associated with hormone replacement therapy in younger and older women: a meta-analysis. J Gen Intern Med. 2004; 19(7):791-804.

6. Salpeter SR, Walsh JM, Greyber E, Salpeter EE. Brief report: coronary heart disease events associated with hormone therapy in younger and older women. A meta-analysis. J Gen Intern Med. 2006;21(4):363-6. 